\title{
A Energy Efficient Approach to DSR based Routing Protocol for Ad Hoc Network
}

\author{
Deepti Badal \\ M.Tech(CSE) \\ Institute of Technology \& Management,Gwalior
}

(M.P.)

\begin{abstract}
The Dynamic Source routing protocol is basically on- demand routing protocol which is used in multi hop wireless network or mesh network. Mobile nodes in Ad hoc network are typically dependent on batteries power. Generally some nodes stop working because battery run out of energy, therefore it is extremely important to conserve the energy. And allready existing route protocol based on Dynamic Source Routing (DSR) suffers from the drawback of energy-consuming. It has major advantages to provide route to data packets to source to destination but its disadvantage is transmission energy which is loss when the data packets are drop or when the acknowledgement not receive by neighbour nodes that time this routes not efficient for data packets. In this situation we give an approach to provide efficient routing which is transmission control approach.
\end{abstract}

\section{Keywords}

DSR Protocol, Route Discovery, Route maintences, TTL, Energy Efficient approach

\author{
Rajendra Singh Kushwah \\ Department of Computer Science \& Engg. \\ Institute of Technology \& Management,Gwalior \\ (M.P.)
}

\section{INTRODUCTION}

The Dynamic Source Routing protocol (DSR) is a simple and efficient routing protocol designed specifically for use in multi-hop wireless ad hoc networks of mobile nodes. DSR allows the network to be completely its self-in structured and organizing, without the need for any existing network infrastructure or administration. Therefore Ad hoc network is applicable to military communications and disaster relief and other special occasions.

Due to the particularity of Ad Hoc network and the dependence of network on routing technology, routing technology has become an issue that in great needs for immediate solution, and it is also the difficulty and emphasis for Ad hoc network research. MANET workgroup of IETF have been focusing on the research of routing protocol for Ad Hoc network, and many protocol draft plans were proposed, such as Dynamic Source Routing protocol (DSR) which is a kind of simple and efficient routing protocol designed for $\mathrm{Ad}$ Hoc network.

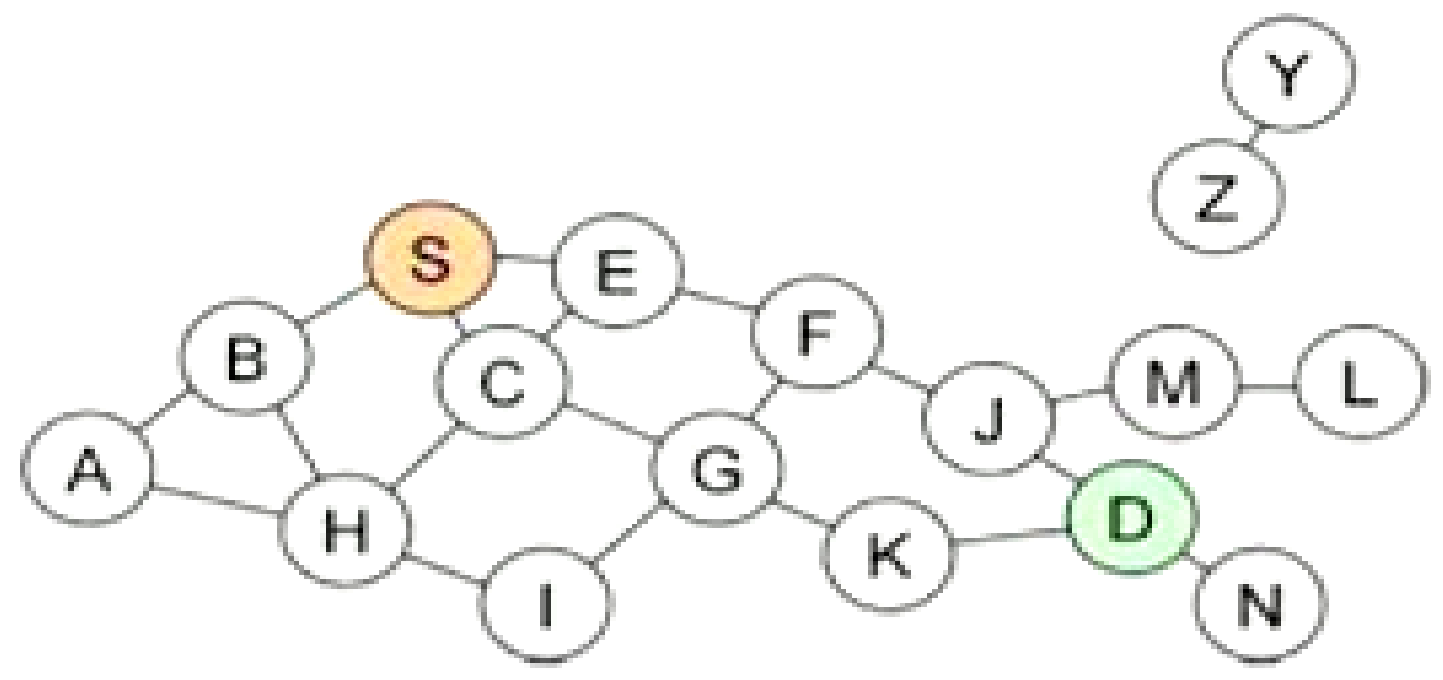

Fig 1:- Message sends from source to destination

This related high performance algorithm behaves well in some highly mobile network. However the mobile nodes on Ad hoc network is generally powered by batteries, the whole performance of network will be downgraded or even lead to the split of the network when energy of some nodes is exhausted. DSR protocol

suffers from energy constraint of each node on network, thus its

effective energy management mechanism for Ad hoc network is necessary [1].

\section{WORKING OF DSR ROUTING PROTOCOL}

The DSR protocol is containing of the two main mechanisms of "Route Discovery" and "Route Maintenance", which work together to allow nodes to discover and maintain routes to destinations in the ad hoc network.

\subsection{Route Discovery}




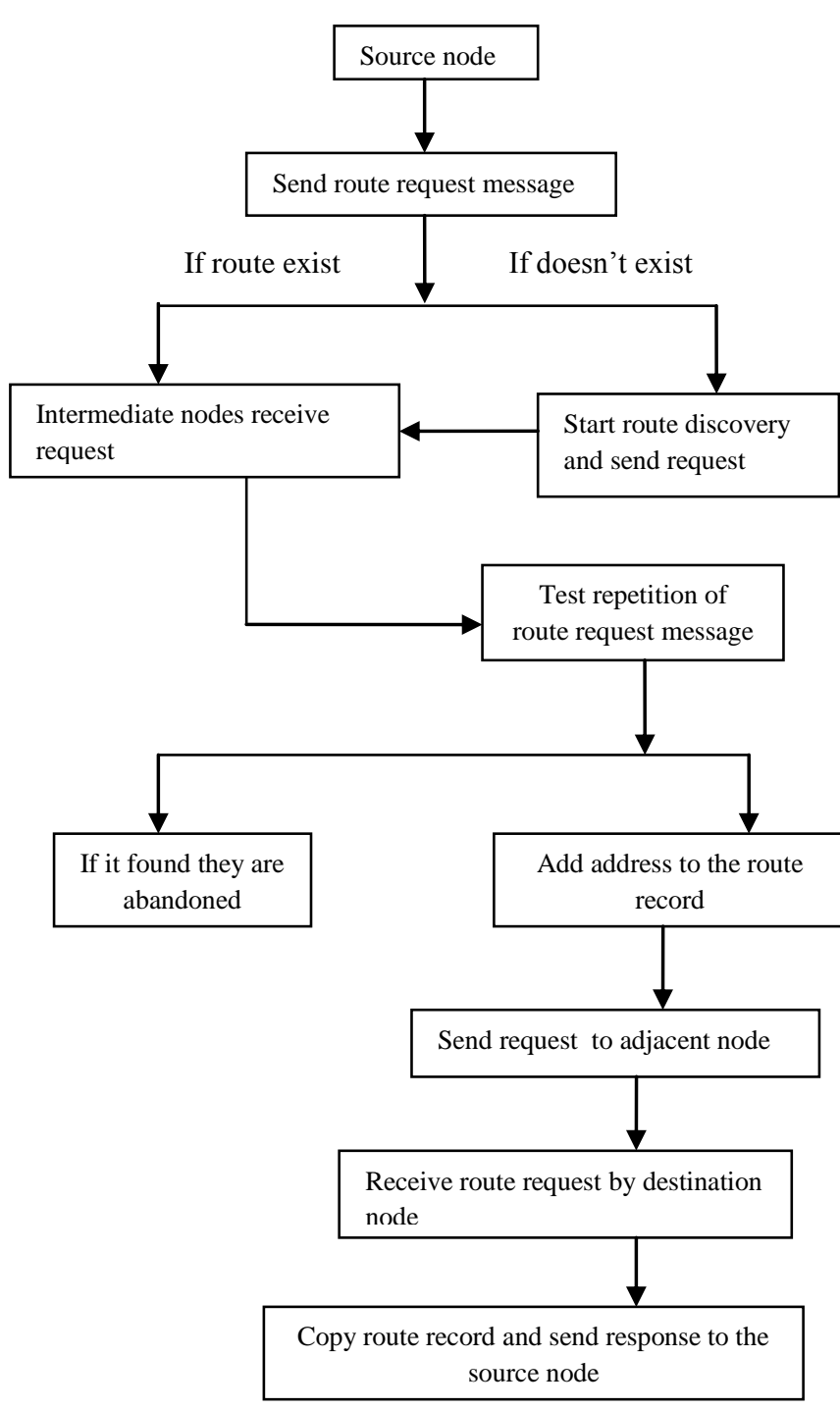

Fig 2:- Process of route discovery

When node $\mathrm{S}$ is to send message to the destination node $\mathrm{D}$, it first queries if there exists route from $S$ to $D$ in the routing buffer. If so, then source node $\mathrm{S}$ sends message according to the route. Otherwise route discovery program is launched, meanwhile source node $\mathrm{S}$ floods route request packet RREQ, When intermediate nodes receive the RREQ message, they test RREQ for the repetition of message.

If repetition of request message is found, they are abandoned, otherwise attach their address to the route record in the head part of packet, and then send this packet to all the neighbour nodes. When the last destination node finally receives RREQ packet, it copies and reverses the route record of the RREQ packet and sends the route reply message RREP to the source nodes, and returns route response message RREP to source node S. Source node buffers the route information locally for future use when receives the RREP packet, this process is shown on the figure 2.That is whole process of route discovery.

\subsection{Route Maintenance}

Once some nodes find the neighbouring link that data is to be sent by is disconnected, they immediately send a route error message RERR to source node $\mathrm{S}$. When the source node receives the error packet, it deletes all the routes that use the invalid link from the buffer, and starts a new route discovery process if necessary. The nodes that forward the error packet along the way delete all the route in the broken link from their own routing table. this process is shown on the figure 3 .

The route discovery procedure of DSR protocol often discovers many routes from source node to destination node. And route with minimal hop is more possible chosen for data transmission than others, the nodes frequently chosen are more likely to consume more energy, which results in short usage of battery.

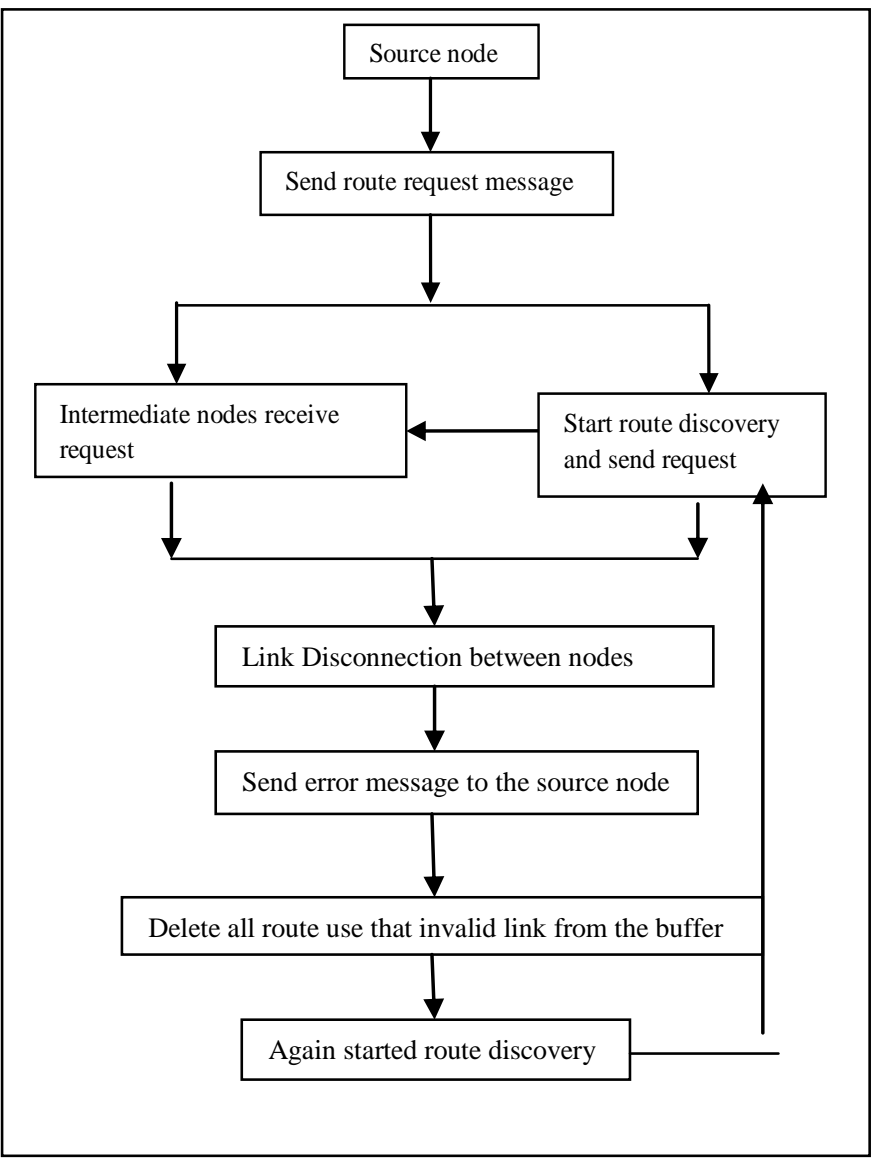

Fig 3:- Process of route maintenances

\section{Advantages:-}

1. Routes are discovered only they are needed

2. Reduces overhead of route maintenance

3 . Route caching reduce the cost of route discovery

4. A single route discovery may yield many routes to the destination, due to intermediate nodes may reply route request from local caches

5. Does not require symmetric links.

Disadvantages:-

1. Packet header size grows with route length due to source routing-Inefficiency

2. Route request packet may potentially reach all nodes in the network-RREQ flooding

3. Route requests may collide at the targeted node -Pay so much but get nothing

4. Every node needs to turn on its receiver all the time-No energy saving

5. Increased contention if too many route replies come back Route Reply Storm 
6. An intermediate node may send Route Reply using a stale cached route, thus polluting other nodes' caches-Mess up routing and forwarding.

\section{RELATED WORK}

Zhenqi Wei et al [2] Server-terminal based distributed speech recognition (DSR) applications are widely adopted on mobile devices. In this paper, we have implemented a powerefficient DSR solution of high performance for realtime speech processing. The DSR frontend algorithms are used to optimize in assembly codes utilizing accelerating techniques provided by a previously released audio DSP, and use as a binary scaling operations in a deep instruction pipeline, automatic memory addressing method, and parallel processing of packaged data for providing energy efficient routing.

Istikmal et al [3] Mobile Ad hoc is a network that does not have the infrastructure and have the ability to manage its network independently, in the future this network process use as a system key to the development of network features. In this paper we use organized routing protocols in mobile ad hoc network (MANET), the optimization is done on the routing protocol DSR (Dynamic Source Routing) which is reactive routing protocol using ant algorithm for analysis and describing the performance of this routing protocol in various scenario and compared the result with standard DSR routing protocol.

G. Rajkumar, R. Kasiram et al [4] the main objective of the paper is to increase the throughput thereby reducing the Network Load and end to end delay between nodes. To achieve this, it is proposed to go for reactive routing protocols. Proactive routing protocols are basically working on table-driven strategy that is the routing tables are exchanged periodically between nodes which lead to more bandwidth and power conservation. In these types of problems, we are depend DSR and AODV protocol. These routing protocols use on- demand system that is the routes are established from source node to destination only on demand which minimizes the delay and packet loss and its throughput. For this process they use "Network Simulator 2.35" the performance of AODV and DSR protocols are compared for large number of nodes in the presence of ambient noise level whereas in the existing works lesser number of nodes is only considered. From our results it is evident that AODV protocol has consume less power compare to DSR and in the presence of high network system, AODV outperforms DSR by yielding higher throughput with less delay.

G. Rajkumar et al [5] the main objective of the paper is to increase the throughput thereby reducing the routing overhead and jitter between nodes. To achieve this, it is proposed to go for reactive routing protocols. Proactive routing protocols are basically table-driven system that is the routing tables which exchanged periodically between nodes which results in more energy consumption. In these problems, we generally go for DSR and AODV.

\section{PROBLEM DEFINITON}

For a wireless networks, the devices operating on battery try to pursue the energy efficiency heuristically by reducing the energy they consumed, while maintaining acceptable performance of certain tasks. Power consumption method is not only a single criterion for measuring and deciding energy efficiency but also measuring by time packet loss and drop. The network can maintain a certain performance level, which is usually said the network lifetime. Hence routing to maximize the lifetime of the network is different from minimum energy routing.

Minimum energy routes [6] sometimes attract more node flows, and the nodes in these routes energy very soon; hence the whole network cannot perform any task due to the network failure on these nodes. In other words, the energy power is balanced consumed among nodes in the networks. Routing with maximum lifetime balances all the routes and nodes globally so that the network maintains certain performance level for a longer time period. Energy efficiency is not only measured and analysis by the power consumption but in more general it can be measured by the duration of time over which the network can maintain a certain performance level.

There are lots of methods for routing algorithms. However, now we distributing them into three parts. One is flooding method and broadcast routing method, which is mostly use during the working of the wireless network, such as to discover node and network failure and broadcast some acknowledgement. The second method is multicast routing, which is use in wireless networks, that is communicate in a one-to-more nodes. The last is unicast, which is worked in an end-to-end node and the most common kind of routing in networks.

Energy is a limiting factor in case of Ad-hoc networks [7][8]. Routing in ad-hoc networks has some unique characteristics. First- energy of nodes is crucial and depends upon battery which has limited power supply. Second-nodes can move in an uncontrolled manner so frequent route failures are possible. Third-wireless channels have lower and more variable bandwidth compare to wired network. Energy efficient routing protocols are the only solution to above conditions. Mostly work is doing on making protocols energy efficient has been done on "on demand routing protocols" because these protocols are more energy efficient rather than on demand protocols. Energy efficiency can achieved flooding at the route discovery process. And energy efficiency can also be achieved by using efficient metric for route selection such as cost function, node energy, battery power etc. Here energy efficiency to provide route from source to destination here energy efficiency means increasing the time duration in which any network maintains certain performance level. We can provide the state of energy efficient routing by increasing the network lifetime and without packet loss and delay.

\section{PROPOSED WORK}

The proposed approach which is very simple and improves energy efficiency without any information about neighbour nodes. In the proposed scheme, when a node receives a RREQ, the node calculates the energy level back off time, which is inversely proportional to the received power of the RREQ. After route discovery, source and intermediate node transmit packets to the destination. There is apply time to Live 
TTL condition. The following steps described the follow of the process.

\author{
Send RREQ to the intermediate node; \\ if $(T T L<=0)$ \\ \{ \\ drop the packet; \\ \} \\ else if $($ Node_id $==$ Dest_id) \\ \{ \\ Consume the RREQ packet; \\ Calculate total delay; \\ Calculate energy level of node on current path; \\ Send RREP to source with best path or active path; \\ \} \\ else \\ \{ \\ Add node id into visited node list; \\ Flood the RREQ packet to his neighbours; \\ \} \\ Respond by the destination node; \\ \} \\ In this scheme we calculate total delay and energy level of the \\ each node and compare the proposed method and DSR \\ protocol. For this process we use network simulator NS2 \\ software which show the performance of network lifetime and \\ consume less energy under the same condition over method \\ DSR. Some basic parameters are use in this process which is \\ packet loss, throughput, end to end delay and energy level of \\ the each node.
}

\section{CONCLUSION}

Aiming at energy-conserving, this paper presents an improved scheme of DSR . The improved method is simple and energysaving routing protocol. The proposed scheme prolongs the lifetime of network without adding to network burden. In a word, the improved protocol is more practical than previous published method. Once a routing system is made, connection by link transmit power adjustment per packet is done based on a energy efficient approach. Energy Efficient approach basically provides a less energy and provides same service to the network. There is a scope to mainly focus on performance analysis of DSR Routing protocol and achieve efficient routing in Ad-hoc network. There is a strong focus on the need a energy efficient routing and its routing management.

\section{REFERENCES}

[1] Linyang Sheng, Jingbo Shao, Jinfeng Ding "A Novel Energy-Efficient Approach to DSR Based Routing
Protocol for Ad Hoc Network” 2010 IEEE.

[2] Zhenqi Wei "Optimization of ETSI DSR frontend software on a high efficient audio DSP", 2013 IEEE.

[3] Istikmal "Analysis and evaluation optimization dynamic source routing (DSR) protocol in mobile AdHoc network based on ant algorithm”, 2013 IEEE.

[4] G. Rajkumar, R. Kasiram "Optimized QoS metrics and performance comparison of DSR and AODV routing protocols", 2012 IEEE

[5] G. Rajkumar "Optimization throughout with reduction in power consumption and performance comparison of DSR and AODV routing protocols", 2012 IEEE

[6] Jangsu Lee, Seunghwan Yoo, and Sungchun Kim, "Energy aware Routing in Location based Ad-hoc Networks," Proceedings of the 4th International Symposium on Communications, Control and Signal Processing, ISCCSP 2010, Limassol, Cyprus, 3-5 March 2010

[7] Dahai Du and Huagang Xiong, "A Location aided Energy-Efficient Routing Protocol for Ad-hoc Networks," in wireless and optical communications conference (WOCC), $201019^{\text {th }}$ annual.

[8] Juan A. Sanchez and Pedro M. Ruiz, “ LEMA: Localized Energy-Efficient Multicast Algorithm based on Geographic Routing," in Proceedings. Of 2006 31st IEEE Conference on Local Computer Networks in 2006.

[9] T. Spyropoulos, K. Psounis. Efficient Routing in Intermittently Connected Mobile Networks: The Multiple-Copy Case, IEEE/ACM transactions on networking, 16(1): 77-90, (2008).

[10] Spyropolulos, K. Psounis, C. S. Raghavendra. Singlecopy Routing in Intermittently Connected Mobile Networks, First Annual IEEE Communications Society Conference on Sensor and Ad Hoc Communications and Networks, pp. 235-244, (2004).

[11] Jingbo Huang,Peilin Hong, Kaiping Xue,A minimum based on energy consumption routing protocol DSR for Mobile Ad Hoc Networks, Journal of circuits and systems, Dec.2008 .

[12] Song Huang, Haitao Bai, Research and Development of Energy Strategy about Ad Hoc, Modern Electronic Technique,2007, 